\title{
Mucus clearance, MyD88-dependent and MyD88-independent immunity modulate lung susceptibility to spontaneous bacterial infection and inflammation
}

\author{
A Livraghi-Butrico ${ }^{1}$, EJ Kelly ${ }^{1}$, ER Klem $^{1}$, H Dang $^{1}$, MC Wolfgang $^{1,2}$, RC Boucher $^{1}$, SH Randell $^{1}$ \\ and WK O’Neal ${ }^{1}$
}

It has been postulated that mucus stasis is central to the pathogenesis of obstructive lung diseases. In Scnn1btransgenic $\left(S c n n 1 b-\mathrm{Tg}^{+}\right)$mice, airway-targeted overexpression of the epithelial $\mathrm{Na}^{+}$channel $\beta$ subunit causes airway surface dehydration, which results in mucus stasis and inflammation. Bronchoalveolar lavage from neonatal Scnn1b$\mathrm{Tg}^{+}$mice, but not wild-type littermates, contained increased mucus, bacteria, and neutrophils, which declined with age. Scnn $1 b-\mathrm{Tg}^{+}$mice lung bacterial flora included environmental and oropharyngeal species, suggesting inhalation and/or aspiration as routes of entry. Genetic deletion of the Toll-interleukin-1 receptor adapter molecule MyD88 in Scnn $1 b-\mathrm{Tg}^{+}$mice did not modify airway mucus obstruction, but caused defective neutrophil recruitment and increased bacterial infection, which persisted into adulthood. Scnn1b- $\mathrm{Tg}^{+}$mice derived into germ-free conditions exhibited mucus obstruction similar to conventional Scnn1b- $\mathrm{Tg}^{+}$mice and sterile inflammation. Collectively, these data suggest that dehydration-induced mucus stasis promotes infection, compounds defects in other immune mechanisms, and alone is sufficient to trigger airway inflammation.

\section{INTRODUCTION}

Respiratory health is maintained by an integrated network of innate and adaptive defense mechanisms. At the airway surface, mechanical mucus clearance is largely responsible for efficient removal of noxious stimuli and mucus stasis has been suggested to produce airway lung diseases, including cystic fibrosis $(\mathrm{CF})$, primary ciliary dyskinesia, and chronic obstructive pulmonary disease (COPD). Effective mucus clearance relies upon multiple processes, e.g., epithelial ion and water transport, mucin secretion, ciliary beat, phasic airway motion, and cough. ${ }^{1}$ Experimentally, mucus stasis has been achieved in mice by airway-targeted overexpression of the epithelial $\mathrm{Na}^{+}$ channel $\beta$ subunit ( $\beta \mathrm{ENaC}$ encoded by the $S c n n 1 b$ gene). ${ }^{2}$ In Scnn1b-transgenic (Scnn1b-Tg ${ }^{+}$mice, airway epithelial $\mathrm{Na}^{+}$ hyperabsorption causes a depletion of airway surface liquid and an increase in mucus concentration, ${ }^{2}$ which is predicted to impair mucus clearance. Indeed, $S c n n 1 b-\mathrm{Tg}^{+}$mice exhibit mucus stasis and airway inflammation, which emerges in the neonatal period and produces a pathology resembling human $\mathrm{CF} /$ chronic bronchitis. ${ }^{2,3}$

Although Scnn $1 b-\mathrm{Tg}^{+}$mice exhibit delayed clearance of instilled bacteria ${ }^{2}$ and chronic neutrophilic inflammation, ${ }^{3}$ the initial studies failed to detect spontaneous bacterial infection, a hallmark of CF lung disease ${ }^{4}$ and an important component of COPD exacerbations. ${ }^{5}$ This observation suggested that mucus stasis could produce inflammation without infection, i.e., sterile inflammation. However, the original microbiological studies were conducted only in adult mice and did not take into account the role of normal developmental processes, including maturation of innate and adaptive immunity ${ }^{6,7}$ and transient abundance of mucous secretory cells, ${ }^{8,9}$ which likely have a role in the evolution of lung pathology in $\mathrm{Scnn} 1 b-\mathrm{Tg}^{+}$mice. Moreover, static airway mucus creates a microaerophilic/hypoxic environment, ${ }^{10}$ which may favor the growth of bacterial species

\footnotetext{
${ }^{1}$ Cystic Fibrosis/Pulmonary Research and Treatment Center, School of Medicine, The University of North Carolina at Chapel Hill, Chapel Hill, North Carolina, USA. ${ }^{2}$ Department of Microbiology and Immunology, The University of North Carolina at Chapel Hill, Chapel Hill, North Carolina, USA. Correspondence: A Livraghi-Butrico (alessandra_livraghi@med.unc.edu) 
not detectable in the conventional aerobic bacterial cultures performed in the original studies of $S c n n 1 b-\mathrm{Tg}^{+}$mice.

Accordingly, we initiated a series of longitudinal studies designed to explore the interactions between mucus clearance and host defense in $S c n n 1 b-\mathrm{Tg}^{+}$mice. First, we tested the hypothesis that neonatal $S c n n 1 b-\mathrm{Tg}^{+}$mice are more susceptible to spontaneous bacterial infection than adult mice, owing to the combination of defective mucus clearance, likely compounded by the transient increase in mucin production that occurs in the early postnatal period, ${ }^{8}$ and immature immune defenses. Thus, we measured bacterial colony-forming units (CFUs) in bronchoalveolar lavage (BAL) as a function of age utilizing bacterial culture conditions that permitted detection of non-fastidious aerobes and microaerophilic bacteria. Second, we hypothesized that mechanical mucus clearance interacts with other developmentally regulated cellular defense mechanisms, e.g., Toll-like receptors (TLRs), ${ }^{11,12}$ which could trigger airway inflammation in response to noxious stimuli accumulating in static mucus. Thus, we longitudinally evaluated whether components of Scnn $1 b$ - $\mathrm{Tg}^{+}$lung pathogenesis were dependent on Tollinterleukin (IL)-1 receptor domain adapter protein myeloid differentiation factor 88 (MyD88), which mediates signaling through several TLRs ${ }^{13}$ and is critical for early postnatal immunity. ${ }^{14,15}$ Finally, to test whether live bacteria were necessary to trigger pulmonary inflammation due to airway surface dehydration and mucus stasis, we generated germ-free (GF) Scnn $1 b-\mathrm{Tg}^{+}$ mice and characterized their lung phenotype.

\section{RESULTS}

\section{Scnn1b-Tg+ mice routinely exhibit spontaneous bacterial infection during the neonatal period but are inflamed throughout life}

The first goal of this study was to determine whether airway surface dehydration and mucus stasis promoted spontaneous bacterial infection in $S c n n 1 b-\mathrm{Tg}^{+}$mice. Longitudinal analysis of BAL from congenic C57BL6/N Scnn1b-Tg ${ }^{+}$and wild-type (WT) littermates revealed no culturable bacteria in WT mice, whereas the majority of $S c n n 1 b-\mathrm{Tg}^{+}$pups harbored intrapulmonary bacteria (Figure 1a). As Scnn $1 b-\mathrm{Tg}^{+}$mice aged, both the proportion of infected mice and the number of bacterial CFUs decreased.

Morphologically distinct bacterial colonies recovered from Scnn $1 b-\mathrm{Tg}^{+}$mice were identified by ribosomal $16 \mathrm{~S}$ gene sequencing (Figure 1b). Scnn $1 b$ - $\mathrm{Tg}^{+}$neonatal lung microflora included both Gram-positive (mainly Streptococcus species and to a lesser extent Staphylococcus xylosus and Rothia nasimurium) and Gram-negative bacteria (mainly Pasturella pneumotropica and Actinobacillus muris). Similar bacterial species were present in tissue homogenates of tongue and esophagus from 5-day-old mice, with no clear distinction between WT or Scnn $1 b-\mathrm{Tg}^{+}$mice (Figure 1c, tongue and esophagus), whereas these bacteria were frequently detected in tracheal homogenates from 5-day-old Scnn $1 b$ - $\mathrm{Tg}^{+}$mice as compared with WT littermates (Figure 1c, trachea). The composition of the lung microflora in adult $S c n n 1 b-\mathrm{Tg}^{+}$mice differed from younger mice, featuring low levels of Gram-positive bacteria,
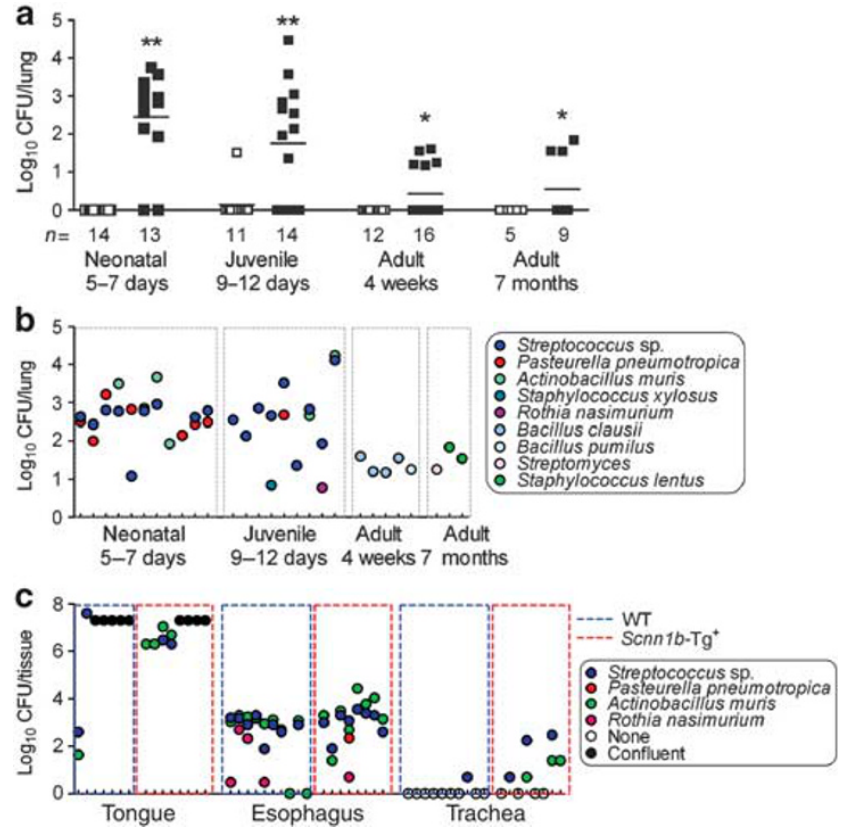

Figure 1 Spontaneous bacterial colonization in C57BL/6N Scnn1btransgenic $\left(S c n n 1 b-\mathrm{Tg}^{+}\right)$mice. (a) Time-course analysis of total colonyforming units (CFUs) in bronchoalveolar lavage samples from Scnn1b$\mathrm{Tg}^{+}$mice ( $\left.\square\right)$ and wild-type (WT) littermates $(\square) .\left(\log _{10}+1\right)$-transformed data; $n=$ number of mice/group; Student's $t$ test ${ }^{\star \star} P<0.005$, ${ }^{\star} P<0.05$ vs. WT littermates. (b) Individual CFUs and bacterial species isolated from C57BL/6N Scnn1b-Tg ${ }^{+}$mice. Each tick on the $\mathrm{x}$ axis represents an individual mouse. (c) Bacteria isolated from tongue, esophagus, and trachea, tissue homogenates of postnatal day (PND) 5 C57BL/6N Scnn $1 b-\mathrm{Tg}^{+}$mice and WT littermates. $\left(\log _{10}+1\right)$-transformed data. Each tick on the $x$ axis represents an individual mouse. CFUs in confluent plates could not be enumerated and were arbitrarily set at $2 \times 10^{7} \mathrm{CFU} /$ tissue.

e.g., Bacillus clausii and Bacillus pumilus at 1 month and Streptomyces and Staphylococcus lentus at 7 months of age (Figure 1b), suggesting a process of selection/adaptation.

Longitudinal analysis of BAL inflammatory markers indicated that $S c n n 1 b-\mathrm{Tg}^{+}$mice exhibited an increased inflammatory infiltrate, predominantly neutrophilic, as compared with WT littermates at all ages (Figure 2). The relative increases in BAL neutrophils, keratinocyte-derived cytokine (KC), and tumor necrosis factor (TNF) $\alpha$ were more robust in neonatal mice (Figure 2b, e, f) when bacterial burden was highest. By contrast, the number of BAL macrophages and lymphocytes in the Scnn $1 b-\mathrm{Tg}^{+}$mice increased with age (Figure 2a, d) and eosinophils peaked at 4-8 weeks (Figure 2c). Macrophages were morphologically activated in both neonatal (5-day-old) and adult (4-week-old) Scnn $1 b-\mathrm{Tg}^{+}$mice, as indicated by higher incidence of enlarged macrophages compared with WT littermates (Figure 2g, h).

\section{Ablation of MyD88 signaling in Scnn1b-Tg+ mice causes defective neutrophil recruitment and higher lung bacterial burden, but does not affect airway mucus obstruction}

The temporal pattern of infection in Scnn $1 b-\mathrm{Tg}^{+}$mice suggests the interaction of airway mucus clearance with other 
a

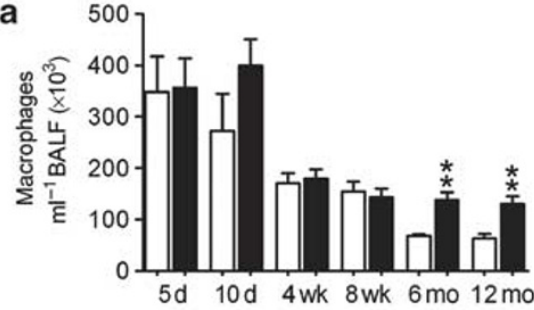

C

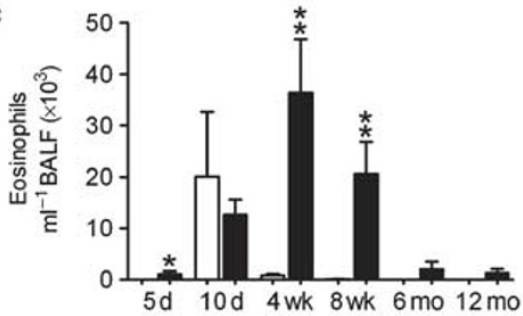

e

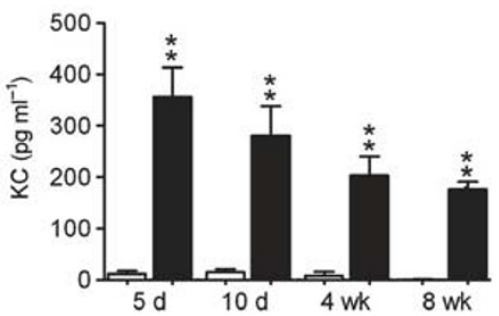

g

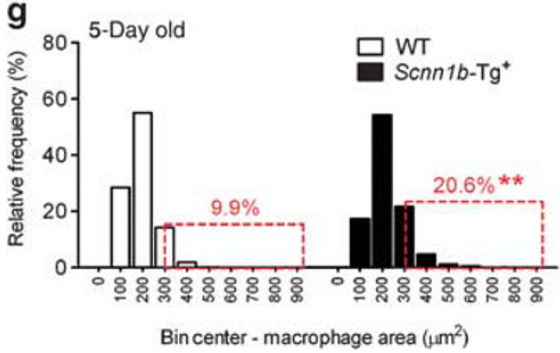

b

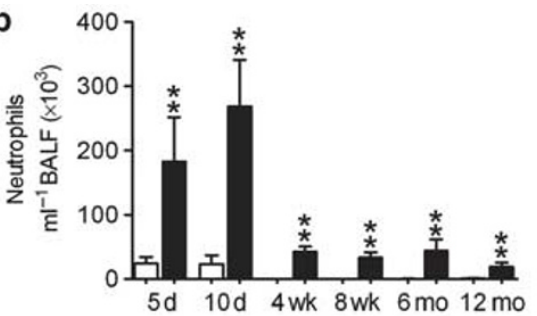

d

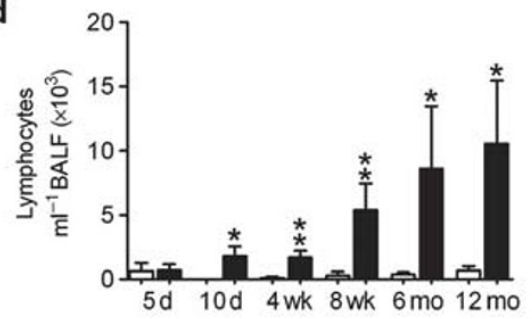

f

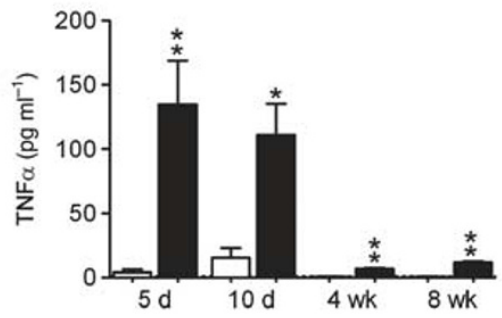

h

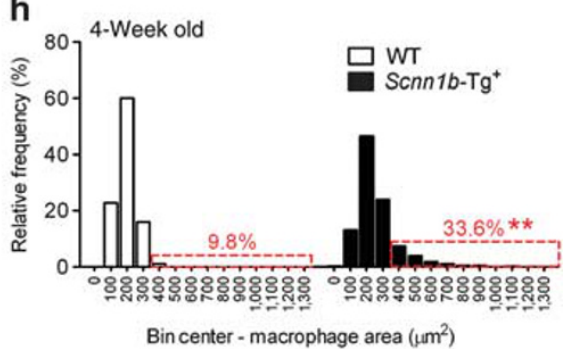

Figure 2 Developmental profile of lung inflammation in C57BL/6N Scnn1b-transgenic (Scnn1b-Tg+) mice. (a-d) Longitudinal bronchoalveolar lavage (BAL) cell counts in Scnn1b-Tg+ mice ( $\square$ ) and wild-type (WT) littermates ( $\square$ ). $n=6$ and 8 at 5 days (5d); $n=7$ and 12 at 10 days (10d); $n=12$ and 13 at 4 weeks (4wk); $n=10$ and 8 at 8 weeks ( $8 \mathrm{wk}) ; n=4$ and 4 at 6 months (6mo); $n=4$ and 6 at 12 months (12mo), for WT and Scnn $1 b$-Tg ${ }^{+}$, respectively. $(e, f)$ Longitudinal keratinocyte-derived cytokine (KC) and tumor necrosis factor $\alpha(T N F \alpha)$ levels in BAL fluid (BALF). $n=4$ WT and 6 Scnn $1 b$-Tg ${ }^{+}$mice. Student's $t$ test ${ }^{* \star} P<0.005,{ }^{*} P<0.05$ vs. WT littermates. (g, h) Macrophage size distribution in 5-day and 4-week-old C57BL/6N Scnn 1b-Tg ${ }^{+}$mice and WT littermates. $n=6$ WT and 8 Scnn $1 b$-Tg ${ }^{+}$mice at postnatal day (PND) $5 ; 10$ WT and 13 Scnn $1 b-\mathrm{Tg}^{+}$mice at PND 28. Boxed regions highlight the percentage of total macrophages larger than the 90 th percentile in WT mice. Student's $t$ test ${ }^{* *} P<0.005,{ }^{\star} P<0.05$ vs. WT littermates.

developmentally regulated innate immune components, e.g., MyD88-dependent TLR signaling. To test this hypothesis, we generated Scnn $1 b-\mathrm{Tg}^{+}$and WT littermates (for this cross, referred to as Scnn1b-Tg-) that were either MyD88-sufficient $\left(\mathrm{MyD} 88^{+/-}\right)$or MyD88-deficient $\left(\mathrm{MyD}^{-18^{-/}}\right)$. When raised under controlled husbandry conditions (see Methods section), survival of all Scnn $1 b-\mathrm{Tg}^{+}$mice ranged between 75 and $100 \%$ (Figure 3a), reflecting the favorable effect of the C57Bl/6N genetic background ${ }^{8}$ compared with the original $\mathrm{C} 3 \mathrm{H}: \mathrm{C} 57$ background, which had $~ 50 \%$ survival. $^{2}$ Importantly, survival of MyD88 $8^{-1-} ; S c n n 1 b-\mathrm{Tg}^{+}$mice was significantly lower than

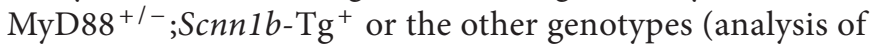
variance, $P=0.004$ ), indicating that combined Scnn $1 b$ transgene overexpression and MyD88 homozygous deletion had a detrimental effect.
Microscopic analysis of BAL cytospin preparations revealed rod- and cocci-shaped bacteria in association with mucus plugs in neonatal MyD88 ${ }^{-/-} ;$Scnn $1 b-\mathrm{Tg}^{+}$mice, which were never observed in MyD88 ${ }^{+/-} ;$Scnn $1 b-\mathrm{Tg}^{+}$mice, despite similar mucus plugs (Figure 3b). Quantification of bacterial burden revealed that $\mathrm{MyD} 88^{-1-} ; \mathrm{Scnn} 1 b-\mathrm{Tg}^{+}$mice harbored significantly more bacteria than MyD88 ${ }^{+/-} ; \mathrm{Scnn} 1 b-\mathrm{Tg}^{+}$mice at all time points and infection persisted into adulthood, although its severity decreased with age (Figure 3c). In particular, heterozygosity for MyD88 was sufficient to protect mice not carrying the Scnn $1 b$ transgene (MyD88 ${ }^{+/-} ; \mathrm{Scnn} 1 b-\mathrm{Tg}^{-}$) from spontaneous bacterial infection. Conversely, complete MyD88 deficiency (MyD88 ${ }^{-/-} ;$Scnn $1 b-\mathrm{Tg}^{-}$) led to significant bacterial burden in neonatal mice, which decreased at postnatal day (PND) 8-10, and was undetectable in adult mice. In agreement with the data 
a

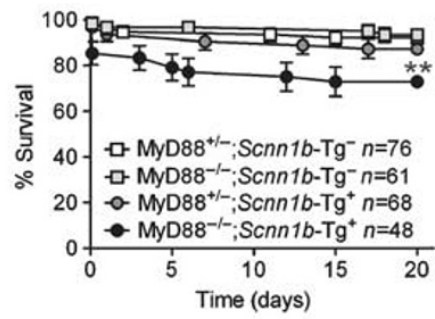

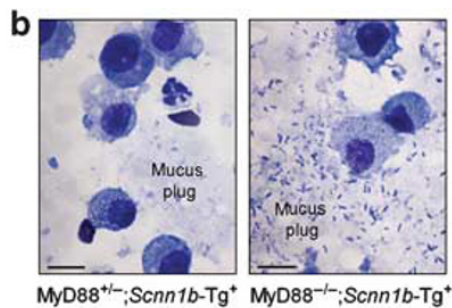

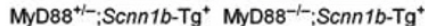
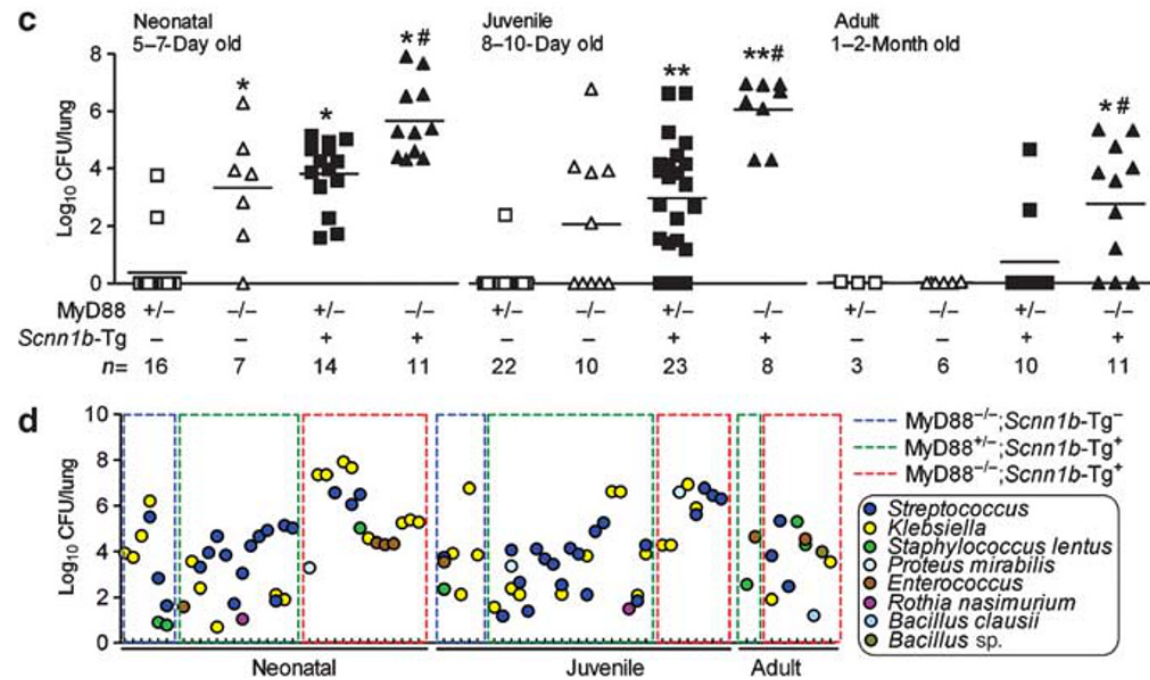

Figure 3 Genetic deletion of myeloid differentiation factor 88 (MyD88) decreases neonatal survival of Scnn1b-transgenic $\left(S c n n 1 b\right.$-Tg $\left.{ }^{+}\right)$mice and increases lung bacterial burden in wild-type (Scnn1b-Tg-) and Scnn1b-Tg ${ }^{+}$mice. (a) Survival curves. Absence of MyD88 in Scnn1b-Tg ${ }^{+}$mice lowers survival. Analysis of variance (ANOVA) ${ }^{*} P=0.004$ vs. littermates. (b) Representative photomicrograph of bronchoalveolar lavage cytospin preparations from neonatal MyD88-sufficient and -deficient Scnn1b-Tg+ mice, illustrating mucus-associated bacteria in MyD88-/-;Scnn1b-Tg+ mice. Bar $=10 \mu \mathrm{m}$. (c) Bacterial colony-forming units (CFUs) in mice from the MyD88 ${ }^{-/} \times S_{\text {Scnn } 1 b-\mathrm{Tg}^{+}}$cross at the ages indicated; (Log $\left.{ }_{10}+1\right)$-transformed

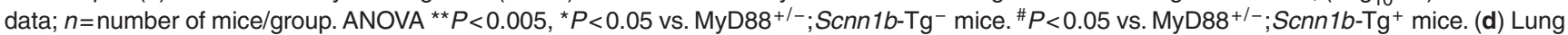
microflora in mice from the MyD88 ${ }^{-/-} \times S c n n 1 b-\mathrm{Tg}^{+}$cross. Each tick on the $\mathrm{x}$ axis represents an individual mouse.

presented in Figure 1a, bacteria were present in BAL of all neonatal MyD88 $8^{+/-} ;$Scnn $1 b-\mathrm{Tg}^{+}$mice and were sporadically detected in adult mice.

The lung microflora of colonized mice from the MyD88 $/$ $\times$ Scnn $1 b-\mathrm{Tg}^{+}$cross (Figure 3d) was also more diverse than in C57BL/6N Scnn $1 b-\mathrm{Tg}^{+}$mice (Figure 1b). Streptococcus and Klebsiella species were prevalent and Staphylococcus, Proteus, Enterococcus, and Rothia species were frequently detected but at lower density (Figure 3d). Notably, Streptococcus, Klebsiella, Staphylococcus, and Enterococcus species persisted in MyD88 ${ }^{-/-} ; \mathrm{Scnn} 16-\mathrm{Tg}^{+}$mice throughout adulthood.

A longitudinal analysis of BAL cell counts and inflammatory mediators was performed to assess the contribution of MyD88 signaling to lung inflammation. Notably, BAL neutrophil counts were lower in MyD88 ${ }^{-/-} ;$Scnn $1 b-\mathrm{Tg}^{+}$vs. MyD88 ${ }^{+/-} ;$Scnn $1 b$ $\mathrm{Tg}^{+}$mice at all time points (Figure $4 \mathbf{b}$ ). Macrophages constituted the predominant cell population in $\mathrm{MyD} 88^{+/-}$and MyD88 $8^{-/-}$mice not carrying the Scnn $1 b$ transgene (Figure 4a). Except for PND 10, when macrophages were increased, Myd88 ${ }^{-1-}$;Scnn $1 b-\mathrm{Tg}^{+}$mice exhibited macrophage numbers (Figure 4a) and morphological activation (Figure 4e) similar to Myd88 ${ }^{+/-} ;$Scnn $1 b-\mathrm{Tg}^{+}$mice. Absence of MyD88 also reduced
BAL eosinophils in 10-day-old Scnn $1 b$ - $\mathrm{Tg}^{+}$mice and prevented the transient developmental eosinophilia in Scnn $1 b-\mathrm{Tg}^{-}$mice $^{8}$ (Figure 4c). Finally, BAL lymphocytes were sharply increased in Myd88 ${ }^{-1-} ;$ Scnn $1 b-\mathrm{Tg}^{+}$mice at PND 10, but waned afterwards (Figure 4d).

The inflammatory mediator profile of cell-free BAL (Figure 5) mirrored the changes in BAL cell composition. Myd88 ${ }^{-I-} ;$ Scnn $1 b-\mathrm{Tg}^{+}$mice exhibited reduced neutrophil chemoattractants (KC, lipopolysaccharide-induced CXC chemokine (LIX), and macrophage inflammatory protein (MIP)-2), granulocyte differentiation factors (granulocyte colony-stimulating factor (G-CSF)), and TNF $\alpha$ at PND 5 compared to Myd88 $8^{+/-}$; Scnn $1 b-\mathrm{Tg}^{+}$mice. Macrophage colony-stimulating factor (M-CSF) was higher at PND 10 in Myd88 ${ }^{-/-} ;$Scnn $1 b-\mathrm{Tg}^{+}$mice, paralleling higher BAL macrophages at this time point. MIP- $1 \alpha$, IL-6, granulocyte macrophage colony-stimulating factor (GMCSF), IL- $1 \alpha$, IL-1 $\beta$, and monocyte chemoattractant protein (MCP)-1 were either at or below the lower limit of detection and differences among groups failed to be significant or exhibit consistent trends (data not shown).

A longitudinal histological analysis was performed to test whether MyD88 deficiency modified the lung pathology in Scnn $1 b-\mathrm{Tg}^{+}$mice (Figure 6). Absence of MyD88 did not alter 

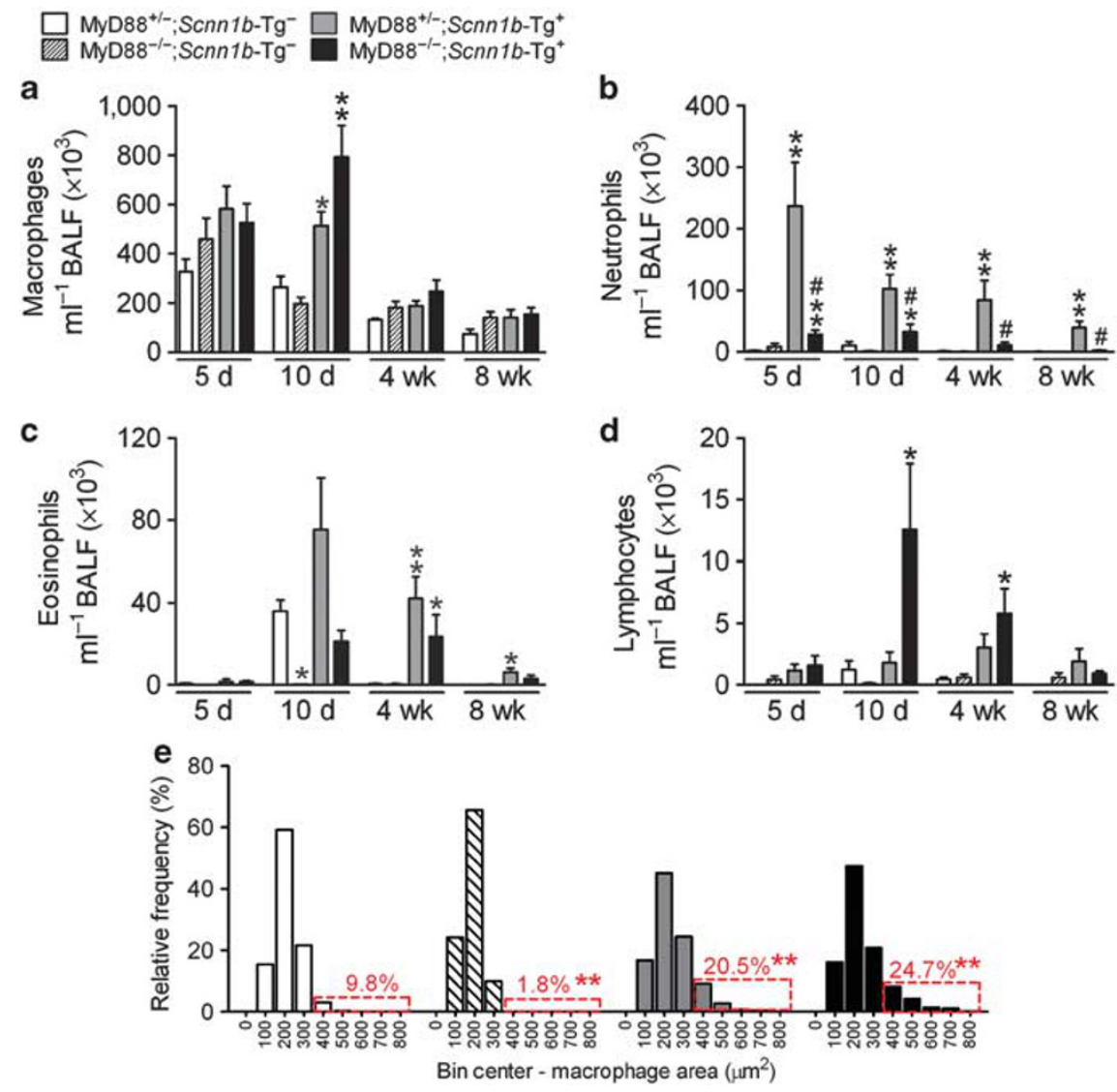

Figure 4 Genetic deletion of myeloid differentiation factor 88 (MyD88) reduces lung neutrophilia in Scnn1b-transgenic $\left(S c n n 1 b\right.$-Tg $\left.{ }^{+}\right)$mice, but does not blunt macrophage activation. (a-d) Differential bronchoalveolar lavage cell counts. $n=8,9,8$, and 8 at 5 days $(5 \mathrm{~d}), n=14,9,12$, and 12

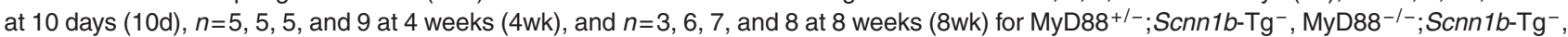
MyD88 ${ }^{+/-} ;$Scnn $1 b-\mathrm{Tg}^{+}$, and MyD88 ${ }^{-/-} ;$Scnn $1 b-\mathrm{Tg}^{+}$mice, respectively. Analysis of variance (ANOVA) ${ }^{* *} P<0.005$, ${ }^{*} P<0.05 \mathrm{vs}$. MyD88 $8^{+/-} ;$Scnn $1 b-$ $\mathrm{Tg}^{-}$mice. ${ }^{\#} P<0.05$ vs. MyD88 ${ }^{+/-} ; S c n n 1 b-\mathrm{Tg}^{+}$mice. (e) Macrophage size distribution in 4-week-old mice from the MyD88 $8^{-/-} \times S c n n 1 b-\mathrm{Tg}^{+} \mathrm{cross}$.

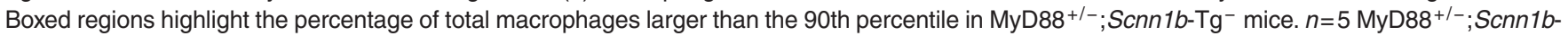

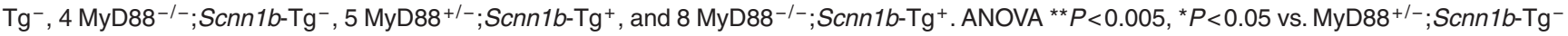
littermates. BALF, bronchoalveolar lavage fluid.

the transient abundance of tracheal and bronchial mucous secretory cells normally observed postnatally ${ }^{8,9}($ Figure 6a, b).

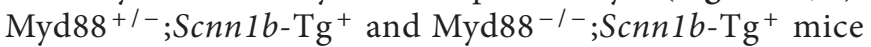
exhibited comparable tracheal mucus plugging and mucous secretory cells in neonates (Figure 6a), a similar progression of mucus obstruction (from trachea to bronchi, Figure 6a, c), and equivalent air space enlargement (data not shown).

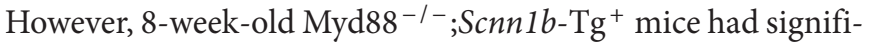
cantly more lymphoid aggregates than $\mathrm{Myd} 88^{+/-} ;$Scnn $1 b-\mathrm{Tg}^{+}$ mice (Figure 6d), suggesting a response to a greater bacterial burden and/or alteration of adaptive immune responses.

\section{Germ-free Scnn1b-Tg+ mice are devoid of bacteria, but still develop mucus obstructive lung disease}

To define the relative contributions of dehydrated mucus vs. neonatal bacterial infection to the development of obstructive airways disease, we derived $S c n n 1 b-\mathrm{Tg}^{+}$mice in a GF environment. As expected, BAL from GF Scnn $1 b-\mathrm{Tg}^{+}$mice harvested at PND 5, when both incidence of infection and bacterial load are maximal in specific pathogen-free (SPF) Scnn $1 b-\mathrm{Tg}^{+}$mice, was sterile $(n=5)$. Adult GF Scnn $1 b-\mathrm{Tg}^{+}$mice exhibited overt airway mucus obstruction and, strikingly, persistent inflammation (Figure 7a-c). Indeed, the overall lung pathology, as assessed by semi-quantitative histology score, was comparable in GF and SPF Scnn $1 b$ - $\mathrm{Tg}^{+}$mice, at both 10 days and 4 weeks of age (Figure $7 \mathbf{d}$ ).

BAL longitudinal analysis revealed that 5-10-day-old GF Scnn $1 b-\mathrm{Tg}^{+}$mice had more macrophages than their WT littermates (Figure 7e), which was not a feature of SPF Scnn $1 b$ $\mathrm{Tg}^{+}$mice (Figure 2a). Although macrophage number in adult GF Scnn $1 b-\mathrm{Tg}^{+}$mice normalized towards WT levels, GF Scnn $1 b-\mathrm{Tg}^{+}$macrophages remained morphologically activated (Figure 7i). GF Scnn $1 b-\mathrm{Tg}^{+}$mice had higher neutrophil counts compared with WT littermates at all time points (Figure 7f) and more eosinophils and lymphocytes in adult mice (Figure $\mathbf{7 g}, \mathbf{h}$ ). Of note, BAL neutrophil counts were lower in neonatal GF $S c n n 1 b-\mathrm{Tg}^{+}$mice compared with SPF Scnn $1 b-\mathrm{Tg}^{+}$mice $\left(87 \pm 18 \times 10^{3}\right.$ vs. $269 \pm 71 \times 10^{3}$ for GF and SPF 10-day-old mice, respectively, $P<0.05$ ), but did not differ in adult mice. As in the SPF environment, the BAL cytokine 

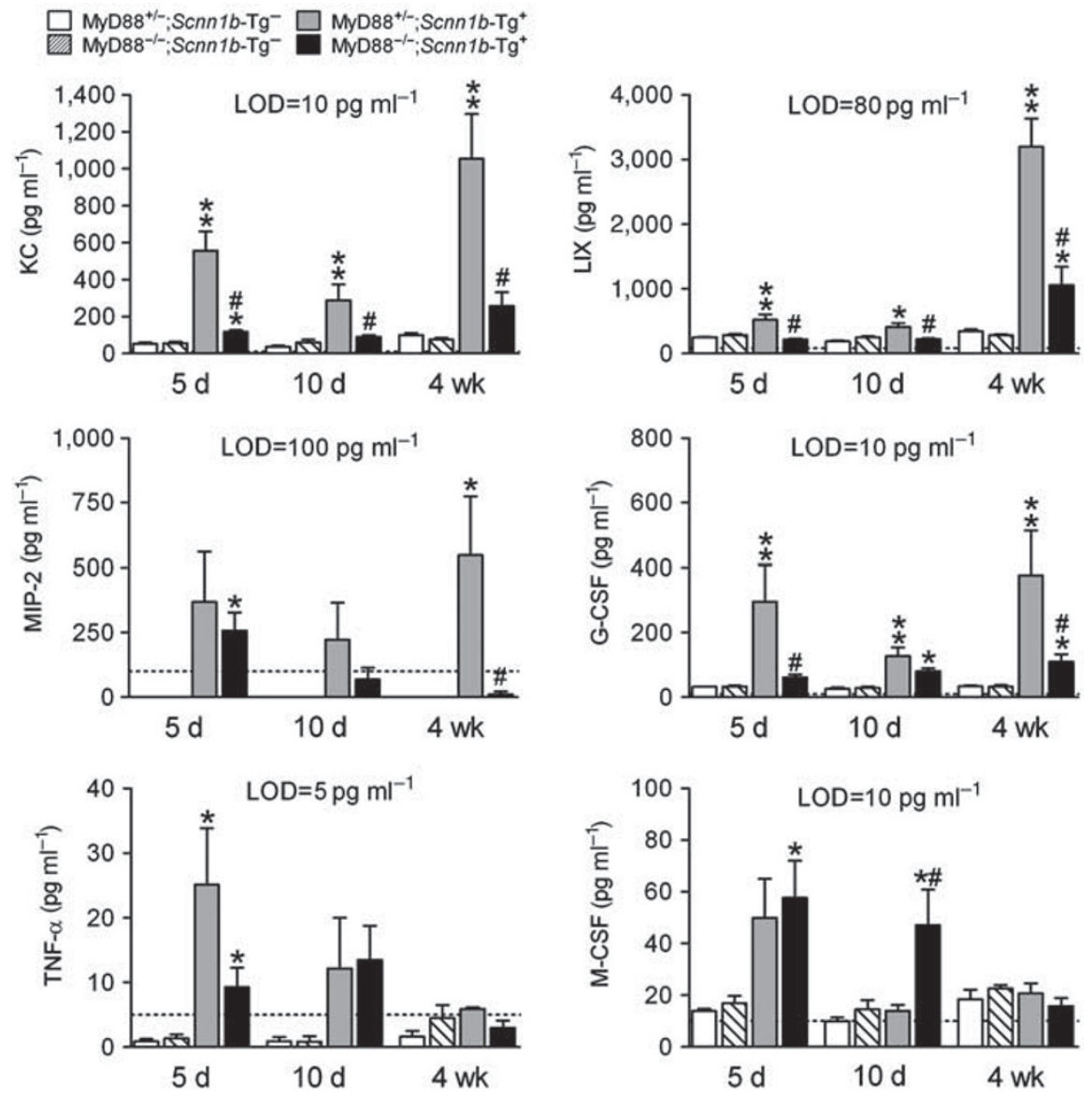

Figure 5 Myeloid differentiation factor 88 (MyD88) deletion in Scnn1b-transgenic (Scnn1b-Tg ${ }^{+}$) mice alters bronchoalveolar lavage (BAL) neutrophil- and macrophage-related inflammatory mediators. BAL cytokines in 5-day-, 10-day- and 4-week-old mice. The dotted line represents the assay lower limit of detection (LOD). $n=4$ for wild-type mice and $n=6$ for Scnn $1 b-\mathrm{Tg}^{+}$mice. Analysis of variance ${ }^{\star \star} P<0.005$, ${ }^{\star} P<0.05$ vs. age-matched MyD88 ${ }^{+/-} ;$Scnn $1 b-\mathrm{Tg}^{-}$mice. ${ }^{\#} P<0.05$ vs. MyD88 ${ }^{+/-} ; S c n n 1 b-\mathrm{Tg}^{+}$mice. G-CSF, granulocyte colony-stimulating factor; KC, keratinocyte-derived cytokine; LIX, lipopolysaccharide-induced CXC chemokine; M-CSF, macrophage colony-stimulating factor; MIP, macrophage inflammatory protein; TNF, tumor necrosis factor.

profile of GF Scnn $1 b-\mathrm{Tg}^{+}$mice was characterized by elevated neutrophil-related mediators (KC, LIX, and G-CSF), whereas, unlike SPF Scnn $1 b-\mathrm{Tg}^{+}$mice, TNF $\alpha$ was not elevated compared with WT littermates (Figure 8a).

Because sterilized feed and bedding in GF conditions are not free of bacterial products, we tested whether environmental lipopolysaccharide (LPS), trapped after inhalation by static airway mucus, could be responsible for stimulating inflammation in GF Scnn $1 b-\mathrm{Tg}^{+}$mice. As expected, the level of LPS in unfractionated BAL from 5-day-old SPF Scnn $1 b$ - $\mathrm{Tg}^{+}$was significantly higher than in WT littermates, but LPS was barely detectable and equivalent in GF Scnn $1 b-\mathrm{Tg}^{+}$mice and WT littermates (Figure 8b). Thus, the inflammation observed in GF Scnn1b$\mathrm{Tg}^{+}$mice appears to be independent of both live bacteria and at least one major bacterial product (LPS).

\section{DISCUSSION}

In the respiratory tract, effective host defense depends primarily upon rapid mechanical clearance of inhaled noxious agents. ${ }^{16}$ The paradigm that defective mucus clearance favors bacterial infections of the airways is widely accepted, ${ }^{1}$ but has never been rigorously tested, nor has the interplay between mucus clearance and other innate defense mechanisms been explored. We used an in vivo model of abnormal mucus clearance, i.e., the Scnn $1 b$ $\mathrm{Tg}^{+}$mouse, to explore the mechanisms involved in acquisition vs. protection from airway bacterial infections.

Spontaneous bacterial infections are rarely reported in mouse models and usually occur in immunocompromized strains. ${ }^{17-19}$ Our longitudinal studies indicate that defective mucus clearance caused a breach in host defense resulting in an agedependent presence of bacteria in the lungs (Figure 1a). This presence likely constitutes an "infection", as indicated by higher neutrophils and TNF $\alpha$ in BAL from SPF Scnn $1 b-\mathrm{Tg}^{+}$mice (Figure $2 \mathbf{b}$ and $\mathbf{f}$ ) compared with GF Scnn $1 b-\mathrm{Tg}^{+}$mice, which have similar mucus obstruction but no bacteria (Figures 7f and 8a). As occurs in $\mathrm{CF},{ }^{10}$ the infection in $S c n n 1 b-\mathrm{Tg}^{+}$mice appears to be centered on the airways (Figure 1c, trachea and Figure $\mathbf{3 b}$, airway mucus). We speculate that the bacterial density $\left(10^{3} /\right.$ lung) likely reprises that observed in human muco-obstructive airway infections. Specifically, we estimated 

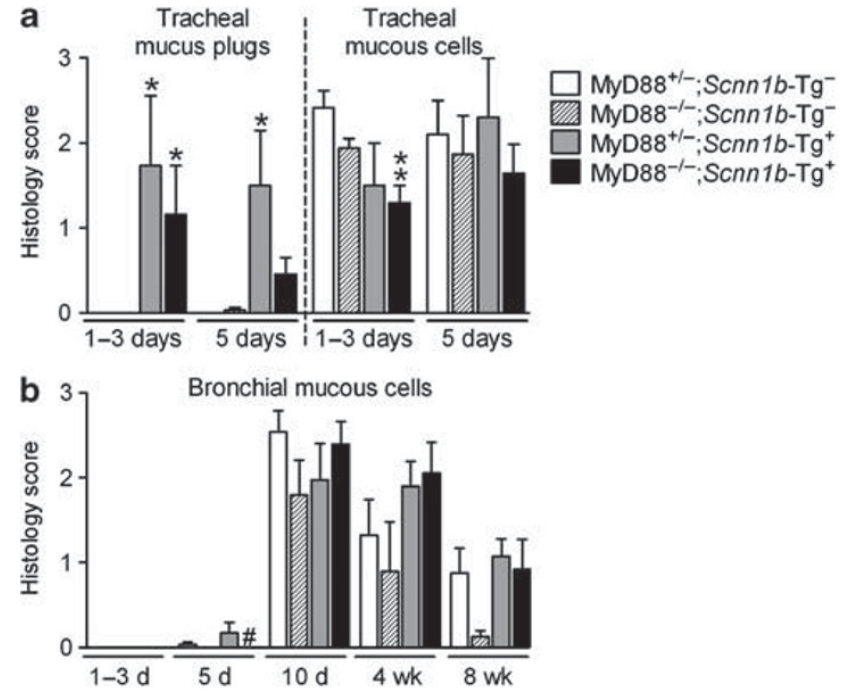

C Bronchial mucus plugs
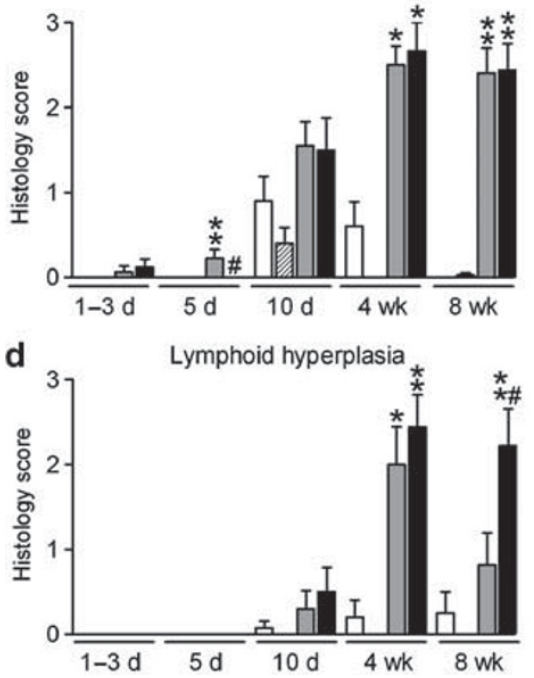

Figure 6 Myeloid differentiation factor 88 (MyD88) deletion does not modify mucus plugging, but promotes development of lymphoid aggregates in Scnn1b-transgenic (Scnn1b-Tg ${ }^{+}$) mice. (a-c) Semiquantitative histopathology scores for mucus plugs and mucous secretory cells (Alcian blue-periodic acid-Schiff positive) in (a) neonatal trachea and (b, c) left lobe intrapulmonary main stem bronchus, at different time points. (d) Semi-quantitative histopathology scores for lymphoid aggregates (bronchial-associated lymphoid tissue). $n=6$, $8,3$, and 5 at $1-3$ days ( $1-3 d), n=5,6,4$, and 8 at 5 days $(5 d), n=13$, 5,10 , and 12 at 10 days (10d), $n=5,4,5$, and 9 at 4 weeks (4wk), and $n=8,8,11$, and 9 at 8 weeks (8wk) for MyD88 ${ }^{+/-} ; S c n n 1 b-\mathrm{Tg}^{-}$; MyD88 ${ }^{-1-} ;$ Scnn1b-Tg ${ }^{-}$; MyD88 ${ }^{+/-} ;$Scnn1b-Tg ${ }^{+}$; and MyD88-/

$-; S c n n 1 b-\mathrm{Tg}^{+}$mice, respectively. Analysis of variance ${ }^{\star *} P<0.005,{ }^{*} P<0.05$ vs. MyD88 ${ }^{+/-} ;$Scnn $1 b-\mathrm{Tg}^{-}$mice. ${ }^{\#} P<0.05$ vs. MyD88 ${ }^{+/-} ;$Scnn $1 b-\mathrm{Tg}^{+}$mice.

that the mucus volume in neonatal Scnn $1 b-\mathrm{Tg}^{+}$mice is $\sim 1 \mu \mathrm{l}$ (see Methods section), producing a density of $10^{6}$ bacteria $\mathrm{ml}^{-1}$, a value typical for sputum samples in CF subjects. ${ }^{20}$

The decrease in lung bacterial burden as $S c n n 1 b-\mathrm{Tg}^{+}$mice aged is likely due to the postnatal maturation of innate ${ }^{6}$ and adaptive $^{7}$ immunity, including changes in pattern recognition receptor expression, ${ }^{11,12}$ neutrophils ${ }^{21}$ and antigen presenting cell functions, ${ }^{22}$ as well as changes in airway mucous secretory cell number/secretions. ${ }^{8,9,23,24}$ Moreover, in mouse airways the density of airway ciliated cells increases with age ${ }^{25}$ and maximal mucus transport rates are generated after PND 9, ${ }^{26}$ partially offsetting the clearance defect owing to mucus dehydration in Scnn $1 b-\mathrm{Tg}^{+}$mice.

The bacterial species most prevalent in the lungs of Scnn $1 b$ $\mathrm{Tg}^{+}$mice during the neonatal period also populated the mouse tongue and esophagus (Figure 1c), suggesting the oropharynx as the portal of entry. We speculate that while aspiration occurred in both WT and Scnn $1 b-\mathrm{Tg}^{+}$mice, infection selectively affected Scnn $1 b-\mathrm{Tg}^{+}$mice due to the poor clearance of aspirated contents. CF patients also exhibit concordant bacterial genotypes in the upper and lower airways, ${ }^{27-29}$ indicating aspiration as a likely mechanism of spread in human diseases associated with pulmonary mucus stasis.

The shift in bacterial species between neonatal and adult Scnn $1 b-\mathrm{Tg}^{+}$mice may indicate that, after clearing the initial infection, Scnn $1 b-\mathrm{Tg}^{+}$mice became susceptible to intermittent infection with species already present in the cage and/or oral environment. Alternatively, bacterial competition in the mucus niche may have favored the species recovered in older mice. Regardless, these data highlight a feature of this model that should be helpful in studying the longitudinal pathogenesis of chronic lung diseases characterized by persistent/recurrent bacterial infection. ${ }^{30}$

Certain caveats apply to our microbiological studies. First, mice housed in SPF facilities are not exposed to highly infectious murine pathogens, which may be more successful in establishing persistent infection in $\mathrm{Scnn} 1 b-\mathrm{Tg}^{+}$mice. Second, BAL sampling might have not harvested all bacteria, but it was preferred over lung homogenization, which was found to inhibit growth of the isolated species. Third, bacterial detection was limited to species capable of growth under the specific culture conditions used, which were not permissive for strict anaerobes and fastidious aerobes. Future studies should focus on exposure to selected pathogens and more sophisticated culture-independent methods suitable to detect small numbers of bacteria, i.e., emulsion PCR and 454 pyrosequencing.

Our studies also demonstrated that other mucosal defense mechanisms modulate the response to airway bacterial infection owing to defective mucus clearance. Studies designed to identify ancillary protective mechanisms revealed that MyD88-dependent innate immunity is key in determining the severity of airway bacterial infections in $S c n n 1 b-\mathrm{Tg}^{+}$mice. In neonatal mice, MyD88 deficiency in the absence of mucus stasis (MyD88 ${ }^{-1-}$;Scnn1b- $\mathrm{Tg}^{-}$mice) produced bacterial burdens comparable to those caused by mucus obstruction alone $\left(\mathrm{MyD} 8^{+/-} ; \mathrm{Scnn} 1 b-\mathrm{Tg}^{+}\right.$mice; Figure 3c), whereas the presence of both MyD88 deficiency and mucus stasis (MyD88 ${ }^{-/-}$; Scnn $1 b-\mathrm{Tg}^{+}$mice) produced an additive effect, suggesting that the two defense mechanisms operate independently. Notably, the greater bacterial burden in neonatal MyD88 ${ }^{-/-}$; 


\section{ARTICLES}

Scnn $1 b-\mathrm{Tg}^{+}$mice compared with MyD88 ${ }^{+/-} ; \mathrm{Scnn} 1 b-\mathrm{Tg}^{+}$ mice was not associated with increased mucus obstruction or mucous secretory cells (Figure 6a-c), indicating that MyD88 deletion predominantly affected the immune functions of inflammatory $^{31}$ and epithelial cells. ${ }^{32}$
The sensitivity of MyD88 $8^{-/-}$mice to bacterial infection differs depending on the microorganism studied, ${ }^{33}$ but is generally regarded as "broad". MyD88 deletion prevents signaling through TLR 1, 2, 4, 5, 7, and 9, and blunts immune responses by impairing neutrophil chemotaxis (Figure $\mathbf{4 b}$ )
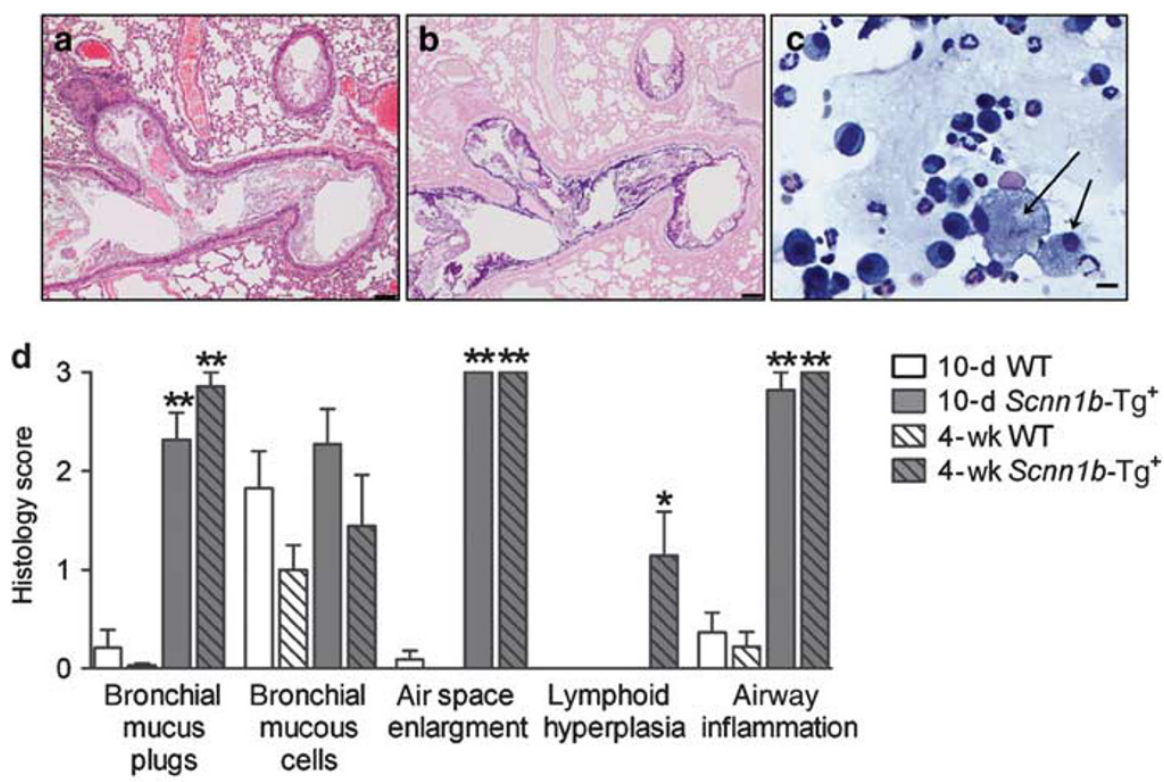

e
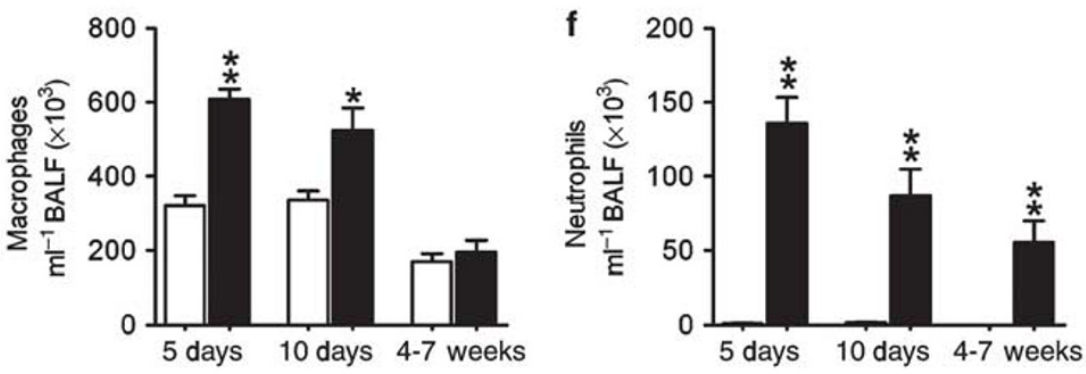

g

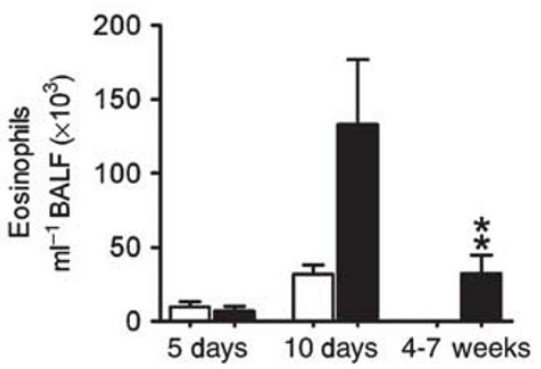

h

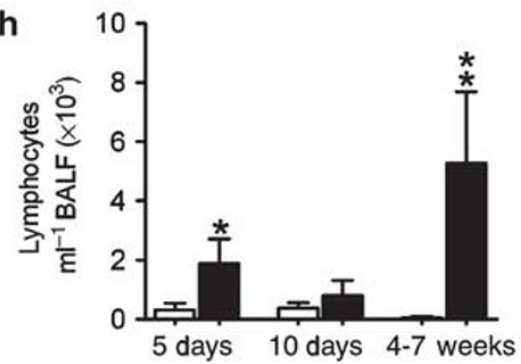

i

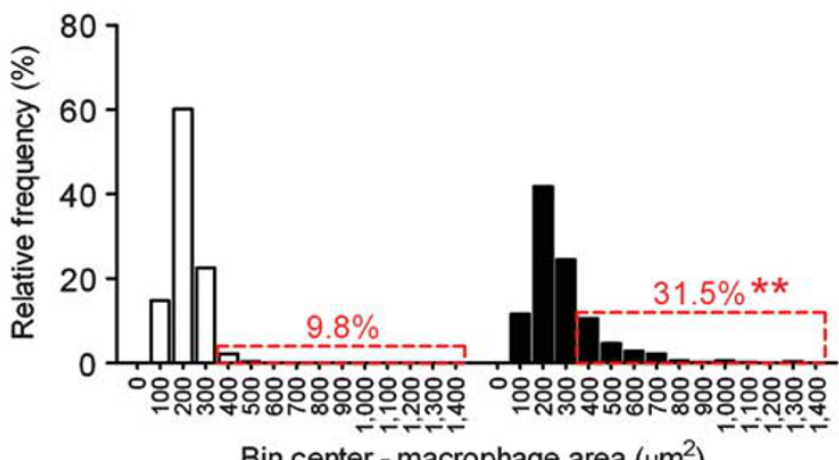


and secretion of inflammatory mediators, such as KC, MIP-2, and CXCL5/LIX (Figure 5). However, both MyD88 ${ }^{-1-}$;Scnn1b$\mathrm{Tg}^{-}$mice and MyD88 ${ }^{-1-} ;$ Scnn $1 b-\mathrm{Tg}^{+}$mice exhibited reduced bacterial burdens as a function of age (Figure 3c), indicating the maturation of MyD88-independent protective mechanisms. ${ }^{13,16,34-36}$ Nonetheless, the higher and persistent bacterial burden in adult MyD88 $8^{-1-} ;$ Scnn $1 b-\mathrm{Tg}^{+}$mice suggests that the MyD88-independent mechanisms were insufficient to completely overcome two additive defects in mucosal defense.

The studies described above highlight the complex interplay between mucus obstruction, infection, and inflammation in our model. The persistence of lung neutrophilia in adult $S c n n 1 b-\mathrm{Tg}^{+}$ mice (Figure 2b) could reflect the presence of non-culturable bacteria. Alternatively, airway mucus stasis per se could contribute to the inflammatory response observed in muco-obstructive lung diseases by impairing clearance of noxious stimuli. To distinguish between these two hypotheses, we generated GF $S c n n 1 b-\mathrm{Tg}^{+}$mice, which were reared in a low-endotoxin environment (Figure 8b). Notably, adult GF Scnn $1 b-\mathrm{Tg}^{+}$mice exhibited lung inflammation and histopathology comparable to SPF Scnn $1 b-\mathrm{Tg}^{+}$mice (Figure 7), supporting the hypothesis that mucus stasis per se can lead to inflammation by trapping non-infectious, noxious materials.

As LPS levels were minimal in the GF environment (Figure 8b), we speculate that other stimuli contributed to macrophage activation (Figure 7i) and neutrophil recruitment (Figure 7f) in GF Scnn $1 b-\mathrm{Tg}^{+}$mice. Beside inhaled noxious stimuli, necrotic bronchial epithelial cells transiently present in 3-day-old Scnn $1 b-\mathrm{Tg}^{+}$mice ${ }^{3}$ could also contribute to the sterile inflammation observed in Scnn $1 b-\mathrm{Tg}^{+}$mice. We speculated that once initiated by poor clearance of toxic materials, the inflammatory process is perpetuated by the retention of stimuli causing macrophage activation and the sequelae of neutrophil recruitment, including secondary necrosis ${ }^{37}$ and release of other chemoattractants, e.g., high-mobility group box 1 and prolineglycine-proline (PGP). ${ }^{38}$

Additional features of mice reared in the GF environment were noted. GF mice still exhibited the transient neonatal surge
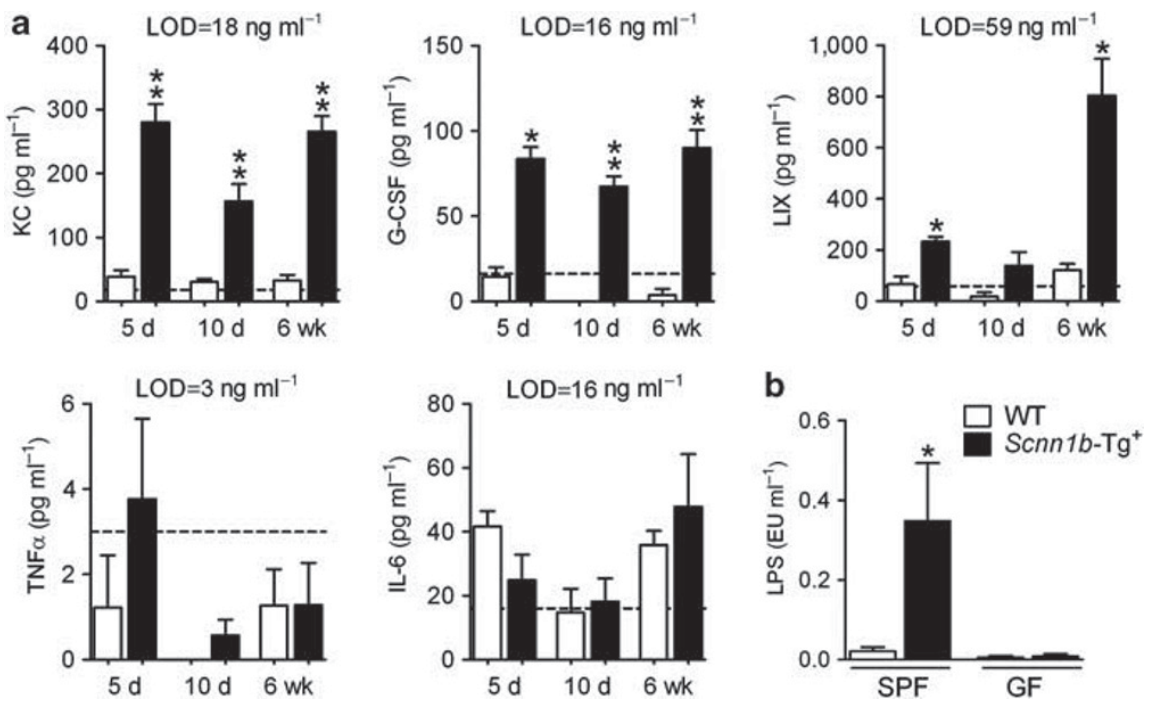

Figure 8 Bronchoalveolar lavage (BAL) cytokine profile and BAL lipopolysaccharide (LPS) content in germ-free (GF) Scnn1b-transgenic $\left(\right.$ Scnn 1 b- $\left.\mathrm{Tg}^{+}\right)$mice and wild-type (WT) littermates. (a) BAL cytokines in 5-day-, 10-day-, and 6-week-old mice. The dotted line represents the assay lower limit of detection (LOD). $n=6$ for WT and 8 for $S c n n 1 b-\mathrm{Tg}^{+}$mice. Analysis of variance ${ }^{\star \star} P<0.005,{ }^{*} P<0.05$ vs. WT littermates. (b) LPS content in BAL isolated from specific pathogen-free (SPF) or germ-free (GF) Scnn $1 b$ - $\mathrm{Tg}^{+}$mice ( $\square$ ) and WT littermates ( $\square$ ). $n=14$ and 11 for SPF WT and $S c n n 1 b-T^{+}$mice, respectively; $n=4$ and 5 for GF WT and Scnn $1 b-T^{+}$mice, respectively. Student's $t$ test ${ }^{\star} P<0.05$ vs. WT littermates. EU, endotoxin unit; G-CSF, granulocyte colony-stimulating factor; IL, interleukin; KC, keratinocyte-derived cytokine; LIX, lipopolysaccharide-induced CXC chemokine; TNF, tumor necrosis factor.

Figure 7 Germ-free (GF) Scnn1b-transgenic (Scnn1b-Tg ${ }^{+}$) mice develop lung inflammation similar to Scnn $1 b$ - $\mathrm{Tg}^{+}$mice raised in conventional specific pathogen-free conditions. (a, b) Representative photomicrographs of left lobe main stem bronchus from 6-week-old GF Scnn 1b-Tg ${ }^{+}$mice, illustrating alveolar space enlargement, mucus obstruction, and airway inflammation. (a) Hematoxylin and eosin and (b) Alcian blue-periodic acid-Schiff stain. Bar $=100 \mu \mathrm{m}$. (c) Representative photomicrograph of bronchoalveolar lavage (BAL) cytospin preparation from GF Scnn $1 b$-Tg ${ }^{+}$ mice, illustrating mucus plugs (light blue), granulocytes and large/foamy macrophages (arrows). Giemsa stain, bar $=20 \mu \mathrm{m}$. (d) Semi-quantitative histopathology scores for 10-day-old (10d, open bars) and 4-week-old (4wk, hatched bars) Scnn1b-Tg ${ }^{+}$mice (gray) and wild-type (WT) littermates (white) raised in GF conditions, $n=11$ Scnn $1 b-\mathrm{Tg}^{+}$and $11 \mathrm{WT}$ littermates at 10 days, $n=7$ Scnn $1 b-\mathrm{Tg}^{+}$and 9 WT littermates at 4 weeks of age. Student's $t$ test ${ }^{* *} P<0.005$, ${ }^{*} P<0.05$ vs. WT littermates. (e-h) Longitudinal differential BAL cell counts for GF Scnn $1 b-T g^{+}$mice $(\square)$ and WT littermates ( $\square$ ). $n=11$ and 11 at 5 days, $n=18$ and 8 at 10 days, $n=8$ and 7 at $4-7$ weeks, for GF WT and GF Scnn $1 b$-Tg ${ }^{+}$mice, respectively. (i) Macrophage size distribution in 4-7 week-old GF Scnn1b-Tg+ mice $(\square, n=7)$ and WT littermates $(\square, n=8)$. Boxed regions highlight the percentage of total macrophages larger than the 90th percentile in WT mice. Student's $t$ test ${ }^{* *} P<0.005$ vs. WT littermates. BALF, bronchoalveolar lavage fluid. 
in mucous secretory cells and eosinophils described for SPF mice $^{8}$ (Figure 7d, g). Furthermore, neonatal GF WT mice had fewer neutrophils (Figure 7f) than neonatal SPF WT mice (Figure 2b), consistent with the notion that normal airways may not be completely sterile and intrapulmonary bacteria help guide immune development. ${ }^{39}$ Indeed, the absence of environmental bacteria might affect the overall immune response to airway mucus stasis, as suggested by the higher number of macrophages recovered in neonatal GF vs. SPF Scnn $1 b-\mathrm{Tg}^{+}$mice (Figures 2a and 7e). Finally, both SPF and GF Scnn $1 b-\mathrm{Tg}^{+}$mice developed bronchial-associated lymphoid tissue (Figure $\mathbf{7 d}$ and ref. 8), which is usually thought to result from TLR stimulation ${ }^{40}$ and whose incidence correlates with worsening airflow obstruction in COPD patients. ${ }^{41}$ The observation that both MyD88 ${ }^{-/-}$; Scnn $1 b-\mathrm{Tg}^{+}$mice and GF Scnn $1 b-\mathrm{Tg}^{+}$mice exhibit lymphoid aggregates suggests that neither MyD88 signaling nor bacterial stimulation is required for this response in the context of airway mucus stasis.

The hypothesis that CF is characterized by intrinsic lung inflammation in the absence of bacterial infection is controversial, ${ }^{42,43}$ and novel animal models have been generated to better understand the critical phases of disease onset and progression. ${ }^{44,45}$ Although we did not perform BAL studies on prenatal Scnn $1 b-\mathrm{Tg}^{+}$mice, whose airways are not exposed to bacteria or bacterial products, previous studies reported that the concentration of KC and MIP-2 in lung homogenates from newborn (PND 1) Scnn 1b- $\mathrm{Tg}^{+}$mice was not different from that of WT littermates, ${ }^{2}$ whereas an inflammatory infiltrate was clearly present in Scnn $1 b-\mathrm{Tg}^{+}$mice just a few days later (PND3), in conjunction with tracheal mucus plugging, ${ }^{3,46}$ suggesting that the inflammatory response develops as a consequence of airway mucus obstruction. Collectively, our data from the GF and SPF Scnn $1 b-\mathrm{Tg}^{+}$mice suggest that mucus stasis per se produces airway inflammation and that mucus stasis results in susceptibility to infection by bacteria aspirated from the oropharynx. Due to its unique phenotype and the amenability to complex genetic and environmental manipulations, the $S c n n 1 b-\mathrm{Tg}^{+}$mouse model has allowed us to probe the interactions of abnormal mucus clearance and other layers of lung defense, e.g., MyD88dependent pathways. Our data suggest that stagnant mucus can initiate both the inflammatory and infectious components of obstructive lung diseases and thus, is a primary therapeutic target. As such, therapies aimed at promoting mucus clearance would provide three interconnected benefits, namely to alleviate inflammation, eradicate infection, and restore immune homeostasis.

\section{METHODS}

Mice. Mice were housed in a SPF facility at the University of North Carolina at Chapel Hill. Congenic C57Bl6/N Scnn1b- $\mathrm{Tg}^{+}$mice were generated by backcrossing C3:B6 Scnn $1 b-\mathrm{Tg}^{+}$mice line $6608^{2}$ with $\mathrm{C} 57 \mathrm{Bl} / 6 \mathrm{~N}$ mice (Taconic, Hudson, NY) for more than 12 generations. Mice were housed in hot-washed, individually ventilated microisolator cages with corn cob bedding, on a 12-hour day-night cycle, fed regular chow, and given water ad libitum. MyD88 $8^{-/-}$mice (B6.129P2Myd $88^{\text {tm1Aki }} 47$ kindly provided by Dr Shizuo Akira, Osaka University, through Dr Jonathan Serody, University of North Carolina at Chapel
Hill), were bred with C57Bl6 Scnn $1 b-\mathrm{Tg}^{+}$mice to obtain experimental animals of four predicted genotypes with expected Mendelian distribution of $25 \%$ each: MyD88 ${ }^{+/-} ;$Scnn $1 b-\mathrm{Tg}^{-}, \mathrm{MyD}^{-1-} 8^{-1}$ Scnn $1 b-\mathrm{Tg}^{-}$,

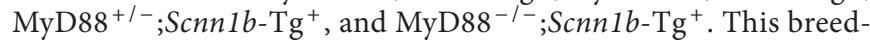
ing strategy generated control mice heterozygous for MyD88, which were not expected to be different from MyD $88^{+/+}$mice, and gave the advantage that experimental animals of all four genotypes were littermates and shared an identical environment. Our first attempt to establish this colony and monitor survival by early toe excision (PND 2-3) was unsuccessful as MyD88 $8^{-1-}$ mice rapidly died, likely owing to susceptibility to infection after early toeing. To increase survival, breeder pairs were housed in "cleaner conditions", i.e., cages with autoclaved TEK-FRESH bedding (Harlan, Indianapolis, IN) changed weekly and were given autoclaved food and antibioticsupplemented water (sulfamethoxazole $0.64 \mathrm{mg} \mathrm{ml}^{-1}$ and trimethoprim $0.13 \mathrm{mg} \mathrm{ml}^{-1}$ ) until the dams gave birth. Pups were toed for identification and genotyping at PND 5. Germ-free Scnn $1 b-\mathrm{Tg}^{+}$mice were generated in the National Gnotobiotic Rodent Resource Center at UNC. Germ-free rodents are axenic, with no detectable bacteria, yeast, molds, parasites, or viruses (except retroviruses). To monitor the sterility of the isolator containing the GF Scnn $1 b-\mathrm{Tg}^{+}$mouse colony, samples of fresh feces, mouth-paws-cages swabs, and drinking water were collected from breeders and holding mice every time the port was open. Collected samples were tested for the presence of contaminating bacteria by plating onto sheep blood agar, growth in fluid thioglycollate medium, and Gram staining. Regardless of port opening, all isolators were periodically monitored (every other month) through Gram staining and 16S PCR on sampled feces. The isolator containing the GF Scnn $1 b-\mathrm{Tg}^{+}$mice colony never suffered a breach in GF status, and we studied mice from different litters at different ages, over a several months period. All animal studies were approved by the Institutional Animal Care and Use Committee of the University of North Carolina at Chapel Hill and performed according to the principles outlined by the Animal Welfare and the National Institutes of Health guidelines for the care and use of animals in biomedical research.

BAL, differential cell counts, lung histology, and macrophage size. For $\geq 10$-day-old mice, we obtained both BAL and lung histology from each animal, as previously described. ${ }^{8}$ Owing to their small size, 5-day-old pups were subject to either whole lung BAL or tissue harvesting. Macrophage size was determined by measuring the surface area of 60-80 macrophages/mouse. Cytospin preparations were photographed with a Leica DMIRB inverted microscope interfaced with a Micro Publisher 3.3 color camera (Q-Imaging, Surrey, BC, Canada) at $\times 20$ magnification and macrophage area was determined using the calibrated freehand selection tool of Image J analysis software (NIH, Bethesda, MD). Histology specimens were scored by an investigator blinded to genotype using a semi-quantitative scoring system, as described previously. ${ }^{8}$

Cytokine profile. Mouse TNF $\alpha, \mathrm{KC}$, MIP-2, MIP-1 $\alpha$, M-CSF, MCP-1, LIX, IL-6, IL-1 $\alpha$, IL-1 $\beta$, GM-CSF and G-CSF were measured in cellfree BAL using a Luminex-based assay (EMD Millipore, Billerica, MA), according to the manufacturer's instructions.

Bacteriology. BAL was performed aseptically, inserting the cannula by tracheotomy in the lower portion of the trachea, to avoid oropharyngeal bacteria. Whole lungs were lavaged four times, using a weightbased formula ${ }^{8}$ and fractions pooled. Serial BAL dilutions were plated onto Columbia anaerobe sheep blood agar (Becton Dickinson, NJ) and incubated in a candle jar to facilitate the growth of microaerophilic bacteria. Plates were incubated at $37^{\circ} \mathrm{C}$ for $24 \mathrm{~h}$, and colony-forming units were enumerated and classified based on their morphology. Individual colonies representative of each morphological group were expanded and processed for molecular identification. For detection of bacteria in tissue homogenates, tongue, esophagus, trachea, and lungs were dissected from 
5-day-old mice and homogenized in $1 \mathrm{ml}$ of sterile Dulbecco's phosphatebuffered saline (Sigma, MO) using a Tissue Tearor (Biospec products, $\mathrm{OK}$ ) on ice. Serial dilutions were plated and processed as described above.

Molecular identification of bacterial species. Bacterial DNA was extracted using the FastDNA SPIN Kit, lysing Matrix B protocol (MP Biomedicals, $\mathrm{OH}$ ), according to the manufacturer's instructions. The ribosomal $16 \mathrm{~S}$ gene was amplified by PCR using high-pressure liquid chromatography-purified primers (forward 5' AGAGTTTGATC(A + C)TGGCTCAG $3^{\prime}$, reverse $5^{\prime}$ TACGG $(\mathrm{C}+\mathrm{T}) \mathrm{TACCTTGTTACGAC}$ TT $3^{\prime}$ ) and the following PCR conditions: denaturing $95^{\circ} \mathrm{C}$ for $5 \mathrm{~min}$; 35 cycles of amplification $\left(95^{\circ} \mathrm{C}\right.$ for $20 \mathrm{~s}, 51^{\circ} \mathrm{C}$ for $20 \mathrm{~s}$, and $72^{\circ} \mathrm{C}$ for $1 \mathrm{~min}$ and $10 \mathrm{~s}$ ); and $72^{\circ} \mathrm{C}$ for $8 \mathrm{~min}$. Primers and deoxyribonucleoside triphosphates were removed from the PCR product by digestion with exonuclease I and shrimp alkaline phosphatase (ExoSAP-IT, USB, $\mathrm{OH}$ ). The PCR product was sequenced using both forward and reverse primers (Genewiz, NJ). Bacterial identities were inferred from the degree of alignment of the ribosomal $16 S$ gene sequences with the existing databases (NCBI Nucleotide collection, excluding "models" and "uncultured/environmental sample sequences"), using the NCBI Basic Local Alignment Search Tool.

LPS assay. Limulus amebocyte lysate assay was performed on unfractionated BAL from 5-day-old WT and Scnn $1 b-\mathrm{Tg}^{+}$mice using the Pyrochrome kinetic method, according to the manufacturer's instructions (Associates of Cape Cod, MA). The assay was performed under conditions that allowed cumulative detection of both LPS and $\beta$-glucan.

Estimate of mucus volume. Mucus volume was calculated from the adult mouse airway surface liquid volume of $2.27 \mu \mathrm{l}^{48}$ and the adult-pup scaling factor of two, obtained by dividing the average tracheal length or diameter in $20 \mathrm{~g}$ vs. $3 \mathrm{~g}$ mice. Measurements were courtesy of Dr Barbara Grubb, University of North Carolina at Chapel Hill, Chapel Hill, NC.

Statistical analyses. Statistical analyses were performed using SigmaStat 3.1 (Aspire Software International, Ashburn, VA) or JMP 8.0. 2 (SAS, Cary, NC). Survival curves were compared using Kaplan-Meier log rank analysis and Holm-Sidak multiple comparison. All numeric values were $\log 10$ transformed with an offset of +1 before inferential statistical analyses. Comparisons between measurements from two groups with significant difference in variances were performed using the Student's $t$ test assuming non-equal variance, or non-parametric Wilcoxon rank-sum test. Comparison between multiple groups was performed using oneway analysis of variance and differences among the group means were assessed by Tukey-Kramer post-hoc test for multiple test correction. For inferential statistics, $P<0.05$ was considered statistically significant and $n$ represents the number of animals in each experimental group. Data presented in plots with error bars are expressed as mean \pm s.e.m. Distribution of macrophage sizes between control (WT or MyD $88^{+/-}$;Scnn $1 b-\mathrm{Tg}^{-}$) and test groups were performed by determining the $90 \%$ threshold in the control group and comparing the proportion of cells in the test group beyond the threshold. Statistical significance between the proportions of cells that passed the threshold in the test group vs. control group (10\%) was evaluated using $\chi^{2}$ test.

\section{ACKNOWLEDGMENTS}

We thank Nanette B. Fulcher for advice in microbiology techniques and data analysis; Kimberly Burns, Donald Joyner, and Tracy Eldred for technical assistance with histology; Kristy Terrell and Kimberly Brassard for assistance with bacterial species identification; Rodney Gilmore for assistance with mouse genotyping; the UNC Michael Hooker Microscopy Facility, funded by an anonymous private donor; the Clinical Proteomics Laboratory at the UNC Thurston Arthritis Research Center and the Immunotechnology Core at the UNC Center for Gastrointestinal Biology and Disease for Luminex assays; and Maureen A. Bower, Kathy Mohr, and Jamison D. Cameron in the UNC Center for Gastrointestinal Biology and Disease Gnotobiotic Core directed by Dr B. Sartor and supported by NIH grant P30 DK34987 for generating and maintaining the germ-free Scnn $1 b-\mathrm{Tg}^{+}$mouse colony. The studies were supported by the grant RANDEL07P0 awarded to S.H.R. by the Cystic Fibrosis Foundation, and by the Cystic Fibrosis Research Development Program grant RDP R026, and National Institute of Health P30 DK065988 and P50 HL060280 to W.K. O'N and R.C. B.

\section{DISCLOSURE}

The authors declared no conflict of interest.

C) 2012 Society for Mucosal Immunology

\section{REFERENCES}

1. Randell, S.H. \& Boucher, R.C. Effective mucus clearance is essential for respiratory health. Am J Respir Cell Mol Biol 35, 20-28 (2006).

2. Mall, M., Grubb, B.R., Harkema, J.R., O'Neal, W.K. \& Boucher, R.C. Increased airway epithelial $\mathrm{Na}+$ absorption produces cystic fibrosis-like lung disease in mice. Nat Med 10, 487-493 (2004).

3. Mall, M.A. et al. Development of chronic bronchitis and emphysema in beta-epithelial $\mathrm{Na}+$ channel-overexpressing mice. Am J Respir Crit Care Med 177, 730-742 (2008).

4. Hauser, A.R., Jain, M., Bar-Meir, M. \& McColley, S.A. Clinical significance of microbial infection and adaptation in cystic fibrosis. Clin Microbiol Rev 24, 29-70 (2011).

5. Sethi, S. Infection as a comorbidity of COPD. Eur Respir J 35, 1209-1215 (2010).

6. Levy, O. Innate immunity of the newborn: basic mechanisms and clinical correlates. Nat Rev Immunol 7, 379-390 (2007).

7. Adkins, B., Leclerc, C. \& Marshall-Clarke, S. Neonatal adaptive immunity comes of age. Nat Rev Immunol 4, 553-564 (2004).

8. Livraghi, A. et al. Airway and lung pathology due to mucosal surface dehydration in \{beta\}-epithelial $\mathrm{Na}+$ channel-overexpressing mice: role of TNF-\{alpha\} and IL-4R\{alpha\} signaling, influence of neonatal development, and limited efficacy of glucocorticoid treatment. J Immunol 182, 4357-4367 (2009).

9. Roy, M.G. et al. Mucin production during pre- and post-natal mouse lung development. Am J Respir Cell Mol Biol 44, 755-760 (2011).

10. Worlitzsch, D. et al. Effects of reduced mucus oxygen concentration in airway Pseudomonas infections of cystic fibrosis patients. J Clin Invest 109, 317-325 (2002).

11. Sadeghi, K. et al. Immaturity of infection control in preterm and term newborns is associated with impaired toll-like receptor signaling. $J$ Infect Dis 195, 296-302 (2007).

12. Al-Hertani, W., Yan, S.R., Byers, D.M. \& Bortolussi, R. Human newborn polymorphonuclear neutrophils exhibit decreased levels of MyD88 and attenuated p38 phosphorylation in response to lipopolysaccharide. Clin Invest Med 30, E44-E53 (2007).

13. Kumar, H., Kawai, T. \& Akira, S. Toll-like receptors and innate immunity. Biochem Biophys Res Commun 388, 621-625 (2009).

14. von Bernuth, $\mathrm{H}$. et al. Pyogenic bacterial infections in humans with MyD88 deficiency. Science 321, 691-696 (2008).

15. Bousfiha, A. et al. Primary immunodeficiencies of protective immunity to primary infections. Clin Immunol 135, 204-209 (2010).

16. Martin, T.R. \& Frevert, C.W. Innate immunity in the lungs. Proc Am Thorac Soc 2, 403-411 (2005).

17. Gozalo, A.S. et al. Spontaneous staphylococcus xylosus infection in mice deficient in NADPH oxidase and comparison with other laboratory mouse strains. J Am Assoc Lab Anim Sci 49, 480-486 (2010).

18. Forlow, S.B., Foley, P.L. \& Ley, K. Severely reduced neutrophil adhesion and impaired host defense against fecal and commensal bacteria in CD18-/-P-selectin-/- double null mice. FASEB J 16, 1488-1496 (2002).

19. Ostanin, D.V., Barlow, S., Shukla, D. \& Grisham, M.B. NADPH oxidase but not myeloperoxidase protects lymphopenic mice from spontaneous infections. Biochem Biophys Res Commun 355, 801-806 (2007).

20. Tunney, M.M. et al. Detection of anaerobic bacteria in high numbers in sputum from patients with cystic fibrosis. Am J Respir Crit Care Med 177, 995-1001 (2008).

21. Garvy, B.A. \& Harmsen, A.G. The importance of neutrophils in resistance to pneumococcal pneumonia in adult and neonatal mice. Inflammation 20, 499-512 (1996). 
22. Garvy, B.A. \& Qureshi, M.H. Delayed inflammatory response to Pneumocystis carinii infection in neonatal mice is due to an inadequate lung environment. J Immunol 165, 6480-6486 (2000).

23. Van Winkle, L.S. et al. Epithelial cell distribution and abundance in rhesus monkey airways during postnatal lung growth and development. J Appl Physio/ 97, 2355-2363; discussion 2354 (2004).

24. Lamb, D. \& Reid, L. Acidic glycoproteins produced by the mucous cells of the bronchial submucosal glands in the fetus and child: a histochemical autoradiographic study. Br J Dis Chest 66, 248-253 (1972).

25. Toskala, E., Smiley-Jewell, S.M., Wong, V.J., King, D. \& Plopper, C.G. Temporal and spatial distribution of ciliogenesis in the tracheobronchial airways of mice. Am J Physiol Lung Cell Mol Physio/ 289, L454-L459 (2005).

26. Francis, R.J. et al. Initiation and maturation of cilia-generated flow in newborn and postnatal mouse airway. Am J Physiol Lung Cell Mol Physiol 296, L1067-L1075 (2009).

27. Muhlebach, M.S. et al. Are lower airway or throat cultures predictive of sinus bacteriology in cystic fibrosis? Pediatr Pulmonol 41, 445-451 (2006).

28. Bonestroo, H.J., de Winter-de Groot, K.M., van der Ent, C.K. \& Arets, H.G. Upper and lower airway cultures in children with cystic fibrosis: do not neglect the upper airways. J Cyst Fibros 9, 130-134 (2010).

29. Mainz, J.G. et al. Concordant genotype of upper and lower airways $P$ aeruginosa and $S$ aureus isolates in cystic fibrosis. Thorax $64,535-540$ (2009).

30. Didierlaurent, A., Goulding, J. \& Hussell, T. The impact of successive infections on the lung microenvironment. Immunology 122, 457-465 (2007).

31. Craig, A., Mai, J., Cai, S. \& Jeyaseelan, S. Neutrophil recruitment to the lungs during bacterial pneumonia. Infect Immun 77, 568-575 (2009).

32. Evans, S.E., Xu, Y., Tuvim, M.J. \& Dickey, B.F. Inducible innate resistance of lung epithelium to infection. Annu Rev Physiol 72, 413-435 (2010).

33. Balamayooran, T., Balamayooran, G. \& Jeyaseelan, S. Review: Toll-like receptors and NOD-like receptors in pulmonary antibacterial immunity. Innate Immun 16, 201-210 (2010).

34. LeBourhis, L. et al. Antimicrobial activity of mucosal-associated invariant T cells. Nat Immunol 11, 701-708 (2010).
35. Ricklin, D., Hajishengallis, G., Yang, K. \& Lambris, J.D. Complement: a key system for immune surveillance and homeostasis. Nat Immunol 11, 785-797 (2010).

36. Zola, T.A., Lysenko, E.S. \& Weiser, J.N. Natural antibody to conserved targets of Haemophilus influenzae limits colonization of the murine nasopharynx. Infect Immun 77, 3458-3465 (2009).

37. Silva, M.T., do Vale, A. \& dos Santos, N.M. Secondary necrosis in multicellular animals: an outcome of apoptosis with pathogenic implications. Apoptosis 13, 463-482 (2008).

38. Rowe, S.M. et al. Potential role of high-mobility group box 1 in cystic fibrosis airway disease. Am J Respir Crit Care Med 178, 822-831 (2008).

39. Hilty, M. et al. Disordered microbial communities in asthmatic airways. PLoS One 5, e8578 (2010).

40. Moghaddam, S.J. et al. Haemophilus influenzae lysate induces aspects of the chronic obstructive pulmonary disease phenotype. Am J Respir Cell Mol Biol 38, 629-638 (2008).

41. Hogg, J.C. et al. The nature of small-airway obstruction in chronic obstructive pulmonary disease. N Engl J Med 350, 2645-2653 (2004).

42. Machen, T.E. Innate immune response in CF airway epithelia: hyperinflammatory? Am J Physiol Cell Physiol 291, C218-C230 (2006).

43. Bruscia, E.M. et al. Macrophages directly contribute to the exaggerated inflammatory response in cystic fibrosis transmembrane conductance regulator-/- mice. Am J Respir Cell Mol Biol 40, 295-304 (2009).

44. Rogers, C.S. et al. Disruption of the CFTR gene produces a model of cystic fibrosis in newborn pigs. Science 321, 1837-1841 (2008).

45. Sun, X. et al. Disease phenotype of a ferret CFTR-knockout model of cystic fibrosis. J Clin Invest 120, 3149-3160 (2010).

46. Wielputz, M.O. et al. In vivo monitoring of cystic fibrosis-like lung disease in mice by volumetric computed tomography. Eur Respir J 38, 1060-1070 (2011).

47. Adachi, O. et al. Targeted disruption of the MyD88 gene results in loss of IL-1- and IL-18-mediated function. Immunity 9, 143-150 (1998).

48. Durairaj, L. et al. Safety assessment of inhaled xylitol in mice and healthy volunteers. Respir Res 5, 13 (2004). 